\title{
Association between Human Papillomavirus Infection and Cytological Abnormalities in Young Female University Students in Central Brazil
}

\section{De Oliveira $\mathbf{D F}{ }^{1}$, Nazareth Pereira $\mathbf{R}^{2}$, Figueiredo Alves $\mathbf{R R}^{4,5}$, Saddi VA², Alves de Sousa NA ${ }^{3}$, Ribeiro $\mathrm{AA}^{2}$, D'Alessandro $\mathrm{WB}^{3}$ and Rabelo Santos $\mathrm{SH}^{1,3 *}$}

${ }^{1}$ Institute of Tropical Pathology and Public Health, Federal University of Goias, Brazil

${ }^{2}$ Pontifical Catholic University of Goiás, Brazil

${ }^{3}$ School of Pharmacy, Federal University of Goiás, Brazil

${ }^{4}$ School of Medicine, Federal University of Goiás, Brazil

${ }^{5}$ School of Medicine, Pontifical Catholic University of Goiás, Brazil

*Corresponding author: Silvia Helena Rabelo Santos, Avenida Universitária c/1ำ Avenida, Setor Universitário, 74605-220, Goiania, GO, Brazil, Tel: +5562 981109616; E-mail: rabelo.silvia@gmail.com

\section{Abstract}

Background: Human papillomavirus (HPV) genital infection is the most common sexually transmitted infection and is acquired when sexual activity begins.

Objective: This study was to evaluate the prevalence and the association between infection with HPV and cytological abnormalities in young female university students in Central Brazil. This study included 200 cervical samples obtained from female university students aged from 18-25 years.

Methods: All specimens were assessed by conventional cytology and tested for 37 HPV genotypes, by using LINEAR ARRAY® HPV Genotyping Test.

Results: HPV-DNA was detected in 94 of 200 female university students (47\%). Cytological abnormalities were detected in $8.7 \%(17 / 196)$ of all satisfactory cervical smears. The results were confirming for Papanicolaou and documented by photomicrographs. HPV 16 was the most commonly detected type in all of cases without cytological abnormalities, followed by HPV 31, 52, 35 and 58. Fifty percent of female university students with a cytological result of LSIL were positive for HPV 16. Other types such as HPV 31, 35, 52 and 58 were detected in cases of ASC-US and ASC-H.

Conclusion: Positivity for any HPV type, including single and multiple infections, was significantly associated with abnormal cervical smear. The high rate of HPV detection in young women emphasizes the advantages of early 
vaccination. The highest prevalence of minor cytological abnormalities justifies the recommendations of cervical cancer screening guidelines.

Keywords: Cervical smears; HPV; Polymerase chain reaction; Young female; Sexually transmitted infection

Abbreviations: HPV: Human Papillomavirus; PCR: Polymerase Chain Reaction; ASC-US: Atypical Squamous Cells of Undetermined Significance; HSIL: High-Grade Squamous Intraepithelial Lesions; UCM: Universal Collection Medium

\section{Introduction}

Human papillomavirus (HPV) genital infection is the most common sexually transmitted infection and is acquired when sexual activity begins [1,2]. Estimates of the prevalence of HPV infection range from $2 \%$ to $44 \%$ around the world $[3,4]$. There are indications that the prevalence is age-dependent and is higher in women under 25 years of age [3,5]. The decrease in the rate of HPV infection with increasing age likely results from some combination of decreased HPV exposure, the selflimiting nature of most infections and a resistance to reinfection $[6,7]$.

Infection of the cervix by one of the oncogenic types of HPV is a prerequisite for the development of invasive cervical cancer $[8,9]$. However, when compared to the prevalence of HPV infection, the rate of lesions that progress to cancer is very low. In young and middleaged women, HPV infections are usually self-limiting. In women in whom regression fails to occur, infection may persist and changes caused by HPV may progress to intraepithelial lesions that are detectable at cytology $[10,11]$.

HPV types that infect the genital tract belong to the family Papillomaviridae, genus Alpha papillomavirus $[12,13]$. Regarding their epidemiological association with cervical cancer and biological studies, $12 \mathrm{HPV}$ types (HPV-16, HPV-18, HPV-31, HPV-33, HPV-35, HPV39, HPV-45, HPV-51, HPV-52, HPV-56, HPV-58 and HPV59) have now been consistently classified as hrHPV (also known as IARC class I). HPV-68 has been classified as probable high-risk (also known as IARC class 2A), and another seven types have been classified as possible high-risk (HPV-26, HPV-53, HPV-66, HPV-67, HPV-70, HPV-73 and HPV-82; also known as IARC class 2B) [14].

The prevalence of cervical cytological abnormalities differs according to the age group and the region studied. In North America, the prevalence of high-grade squamous intraepithelial lesions (HSIL) generally peaks in women less than 30 years of age prior to decreasing in older age groups. In Europe, Middle east and in Central and South America the peak incidence occurs in older women (between the ages of 25 and 35 years) compared to those in North America, before decreasing thereafter. The prevalence of low-grade squamous intraepithelial lesions (LSIL) generally declines with age after a peak in younger age groups (20 to 30 years of age) [15].

HPV positivity rates may vary according to the cytological abnormality detected, particularly in the case of women with atypical squamous cells of undetermined significance (ASC-US) and LSIL. Overall, the prevalence of HPV DNA in cases of cervical cancer is estimated to be approximately $100 \%[3,8,9,16]$. The types most common detected, in descending order of frequency, are HPV 16, 18 and 45 [16]. The prevalence of HPV in HSIL is estimated to be over 85\%, with HPV 16,31 and 58 the most prevalent types [16,17]. In LSIL, the rate of HPV DNA detection may vary from $29 \%$ to $100 \%$, possibly as a function of the cytological procedure and the sensitivity of polymerase chain reaction (PCR) assays. The most frequently detected types are HPV 16, 31 and 51 [16]. Finally, in cases of ASC-US, HPV positivity varies according to the age group, decreasing from $78 \%$ in the 18-24-year age group to $32 \%$ in women over 35 years of age. HPV 16 is the most prevalent type in those groups [16].

Young women are exposed to HPV infection more often because of their sexual behavior, and are more vulnerable than older women because the transformation zone is located on the ectocervix (cervical ectopy) $[18,19]$. The primary target cells for infection may be cells close to the squamo-columnar junction such as the epithelial reserve cells, which lie immediately underneath the columnar epithelium of the endocervix, and which eventually form the stratified epithelial layers of the transformation zone as the cervix matures. For some time now, the general hypothesis has been that lesion formation begins with the infection of a basal stem cell (rather than a basal transiently amplifying cell) and that the longevity of the stem cells is a key factor in the formation of a persistent lesion. For the low-risk HPV types, which do not generally cause neoplasia and which do not massively stimulate basal cell proliferation, this is a plausible hypothesis, even though not yet formally proven [20]. 
There are limited data available on the prevalence and association between HPV infection and cytological abnormalities in young female university students. Therefore, the objective of this study was to evaluate the prevalence and the association between different HPV types and cytological abnormalities in young female university students from a region in Central Brazil.

\section{Material and Methods}

\section{Patients and Sampling}

Cervical samples were obtained from 200 female university students aged from 18-25 years (mean age 21.89 years). The study was conducted in Central Brazil between September 2008 and April 2010. Cervical samples were collected from all participants. After preparing a conventional smear, the cytobrush was placed in a vial containing Universal Collection Medium (UCM) for HPV testing. The study protocol was approved by the institutional review board, and an informed consent was signed by all the women participating in the study.

\section{Cervical Cytology Assessment}

Conventional cytology smears were screened, and the rapid rescreening of all negative cytology smears was performed as a quality control measure [21]. The adequacy of the samples and the degree of abnormalities were classified in accordance with the 2001 Bethesda System [22].

\section{DNA Extraction}

DNA extraction was performed by using a purification kit for the selective binding of DNA to a silica-based membrane in the presence of chaotropic salts (PureLink ${ }^{\mathrm{T}}$, Invitrogen, and Carlsbad, CA, USA). Firstly, $20 \mu \mathrm{L}$ of proteinase $\mathrm{K}$ and $20 \mu \mathrm{L}$ of ribonuclease (RNase) were added to aliquots of $200 \mu \mathrm{L}$ UCM and incubated at room temperature for 2 minutes. Next, 200 $\mu \mathrm{L}$ lysis/binding buffer were added and incubated at $55^{\circ} \mathrm{C}$ for 30 minutes. Lysis was then followed by the addition of $200 \mu \mathrm{L}$ of ethanol $96-100 \%$ and vigorous vortex mixing to yield a homogenous solution. The lysate was applied to the spin column and the purification procedure was performed according to the instructions provided by the manufacturer. The extracted DNA was then stored at $-20^{\circ} \mathrm{C}$.

\section{HPV Amplification and Genotyping}

HPV amplification and genotyping was carried out by using the Roche polymerase chain reaction (PCR)-based LINEAR ARRAY® HPV genotyping test (Roche
Molecular Systems, Branchburg, NJ, USA). HPV DNA was amplified using the L1 consensus biotinylated PGMY09/PGMY11 primer set in a thermal cycler at $95^{\circ} \mathrm{C}$ for 13 minutes, followed by denaturation for 1 minute at $95^{\circ} \mathrm{C}$, annealing for 1 minute at $55^{\circ} \mathrm{C}$, and extension at $72^{\circ} \mathrm{C}$ for 1 minute for a total of 40 cycles. Amplification was followed by a 5 -minute terminal extension step at $72^{\circ} \mathrm{C}$. Biotinylated $\mathrm{GH} 20$ and PC04 primers to the $\beta$ globin gene were used as amplification control. PCR products were denatured in $1.6 \%$ sodium hydroxide $(\mathrm{NaOH})$ and hybridized to an immobilized probe array containing probes for $\beta$-globin at 2 concentrations plus 19 HR-HPV and 18 LR-HPV genotypes. The HR-HPV group included HPV types $16,18,26,31,33,35,39,45$, $51,52,53,56,58,59,66,68,69,73$ and 82 . The LR-HPV group included types $6,11,40,42,54,55,61,62,64,67$, 70, 71, 72, 81, 83, 84, IS39 and CP6108. Positive hybridization was detected by streptavidin-horseradish peroxidase-mediated color precipitation on the membrane at the probe array.

\section{Statistical Analysis}

The detection rates of HPV genotypes in single or multiple infections were distributed according to the cytological findings. The associations between HPV infection and the cytological outcome were assessed using odds ratios (OR) with their respective 95\% confidence intervals $(95 \% \mathrm{CI})$. Two-tailed p-values < 0.05 were considered statistically significant. The entire statistical analysis was carried out using the SAS software package, version 8.0.

\section{Results}

HPV infection was detected in 94 of the 200 female university students (47\%). Infections with HR-HPV genotypes were more common $(77.7 \%$; 73/94) than LRHPV infections $(22.3 \% ; 21 / 94)$.The rate of single infection was $45.7 \%$ (43/94), while multiple infections represented $54.3 \%$ (51/94) of all HPV-positive cases. Overall, 196 of the 200 cytology smears were considered satisfactory for cytology analysis. Cytological abnormalities were detected in $8.7 \%$ of all satisfactory cervical smears (17/196).

Table 1 shows the age-standardized distribution of positive and negative HPV cases in accordance with the cytological results. When valid cytological outcomes are taken into consideration, HPV-DNA was found in 45.7\% $(16 / 35)$ of the university students under 20 years of age and in $47.2 \%(76 / 161)$ of the students in the $20-25$ year age group. Cytological abnormalities were detected in three of the 35 students less than 20 years of age and in 14 of the 161 participants aged 20 to 25 years. 


\begin{tabular}{|c|c|c|c|c|c|}
\hline & \multicolumn{5}{|c|}{ HPV detection } \\
\hline & \multicolumn{2}{|c|}{ Negative } & \multicolumn{2}{|c|}{$\begin{array}{l}\text { Positive } \\
\end{array}$} & \multirow[t]{2}{*}{ Total } \\
\hline & $\mathbf{n}$ & $\%$ & $\mathbf{n}$ & $\%$ & \\
\hline \multicolumn{6}{|c|}{ Cytological Results } \\
\hline \multicolumn{6}{|c|}{$\leq 19$ years } \\
\hline Normal & 19 & 54.3 & 13 & 37.1 & 32 \\
\hline ASC-US & 0 & 0 & 1 & 2.9 & 1 \\
\hline ASC-H & 0 & 0 & 1 & 2.9 & 1 \\
\hline LSIL & 0 & 0 & 1 & 2.9 & 1 \\
\hline Total & 19 & 54.3 & 16 & 45.7 & 35 \\
\hline \multicolumn{6}{|c|}{ 20-25 years } \\
\hline Normal & 80 & 49.7 & 67 & 41.6 & 147 \\
\hline ASC-US & 5 & 3.1 & 5 & 3.1 & 10 \\
\hline ASC-H & 0 & 0 & 1 & 0.6 & 1 \\
\hline LSIL & 0 & 0 & 3 & 1.9 & 3 \\
\hline Total & 85 & 52.8 & 76 & 47.2 & 161 \\
\hline
\end{tabular}

Table 1: Age-standardized HPV-positive and negative cases in accordance with age group and cytological results.

The distribution of HPV genotypes according to the cytological results is shown in (Table 2). The rate of overall HPV infection was $45.1 \%(80 / 179)$ in students with normal cytology and $70.6 \%$ (12/17) among students with abnormal cervical cytology. HPV 16 was the most commonly HR-HPV type detected in all of cases without cytological abnormalities (10\%; 18/179); followed by HPV 31 (4.5\%; 8/179), HPV 52 (3.9\%; 7/179), HPV 35 and HPV 58 (2.2\%; 4/179) as single and multiple infections. HPV 16 was found in 50\% (2/4) of students with a cytological result of low-grade squamous intraepithelial lesion (LSIL). HPV 58, as single and multiple infections, was detected in $18.2 \%$ $(2 / 11)$ of cases of atypical squamous cells of undermined significance (ASC-US) and 50\% (1/2) of results interpreted as atypical squamous cells cannot exclude high-grade squamous intraepithelial lesion (ASC-H). HPV 31 in multiple infections was found in $9.1 \%(1 / 11)$ of cases of ASC-US and in 25\% (1/4) of results interpreted as LSIL. Type 35 as a multiple infection was detected in $9.1 \%(1 / 11)$ of results of ASCUS. HPV 52 as single infection was found in $50 \%(1 / 2)$ of suggestive results of ASC-H.

\begin{tabular}{|c|c|c|c|c|c|}
\hline \multicolumn{7}{|c|}{ Cytological Results } \\
\hline Types & $\begin{array}{c}\text { Negative } \\
\mathbf{n}(\%)\end{array}$ & $\begin{array}{c}\text { ASC-US } \\
\mathbf{n}(\%)\end{array}$ & $\begin{array}{c}\text { ASC-H } \\
\mathbf{n}(\%)\end{array}$ & $\begin{array}{c}\text { LSIL } \\
\mathbf{n}(\%)\end{array}$ & $\begin{array}{c}\text { Total } \\
\mathbf{n}(\%)\end{array}$ \\
\hline 16 & $5(2.8)$ & - & - & - & $5(2.5)$ \\
\hline 16 and others & $13(7.3)$ & - & - & $2(50)$ & $15(7.7)$ \\
\hline 18 & $1(0.6)$ & - & - & - & $1(0.5)$ \\
\hline 18 and others & $2(1.1)$ & - & - & - & $2(1)$ \\
\hline 31 & $3(1.7)$ & - & - & $1(25)$ & $7(1.5)$ \\
\hline 31 and others & $5(2.8)$ & $1(9.1)$ & - & - & $3(1.5)$ \\
\hline 35 & $3(1.7)$ & - & - & - & $2(1)$ \\
\hline 35 and others & $1(0.6)$ & $1(9.1)$ & - & - & $5(2.5)$ \\
\hline 52 & $4(2.2)$ & - & $1(50)$ & - & $3(1.5)$ \\
\hline 52 and others & $3(1.7)$ & - & - & - & $2(1)$ \\
\hline 58 & - & $1(9.1)$ & $1(50)$ & - & $1(2.5)$ \\
\hline 58 and others & $4(2.2)$ & $1(9.1)$ & - & - & $1(0.5)$ \\
\hline 39 & $1(0.6)$ & - & - & - & $1(0.5)$ \\
\hline 39 and others & $1(0.6)$ & - & - & - & $2(1)$ \\
\hline 45 & $1(0.6)$ & - & - & - & $2(1)$ \\
\hline 51 & $2(1.1)$ & - & - & - & $1(0.5)$ \\
\hline 51 and others & $2(1.1)$ & - & - & - & - \\
\hline 66 & $1(0.6)$ & - & - & & - \\
\hline
\end{tabular}




\section{Cytology \& Histology International Journal}

\begin{tabular}{|c|c|c|c|c|c|}
\hline 66 and others & $4(2.2)$ & - & - & - & $4(2)$ \\
\hline 53 and others & $2(1.1)$ & - & - & - & $2(1)$ \\
\hline 56 & $2(1.1)$ & & & & $2(1)$ \\
\hline 56 and others & $1(0.6)$ & - & - & - & $1(0.5)$ \\
\hline 68 & $1(0.6)$ & - & - & - & $1(0.5)$ \\
\hline Low-risk & $18(10.1)$ & $2(18.2)$ & - & $1(25)$ & $21(10.7)$ \\
\hline HPV positive & $80(44.7)$ & $6(54.5)$ & $2(100)$ & $4(100)$ & $92(46.9)$ \\
\hline HPV negative & $99(55.3)$ & $5(45.5)$ & - & - & $104(53.1)$ \\
\hline Total & $179(100)$ & $11(100)$ & $2(100)$ & $4(100)$ & $196(100)$ \\
\hline
\end{tabular}

Table 2: HPV genotypes distribution in cytological results.

Table 3 shows the correlation between abnormal cervical cytology and infection with any type of HPV or HR-HPV. Positivity for any HPV type, including single and multiple infections, was significantly associated with an abnormal cervical smear $(\mathrm{OR}=2.97$; $95 \% \mathrm{CI}$ 1.004-8.781). However, when only infections with HRHPV genotypes were taken into consideration, no statistically significant association was found with abnormal cervical cytology $(\mathrm{OR}=2.12$; $95 \% \mathrm{CI} 0.78-$ 5.78).

\begin{tabular}{|c|c|c|}
\hline HPV infection & OR (95\%CI) & p-value \\
\hline $\begin{array}{c}\text { Infection with any } \\
\text { HPV type }\end{array}$ & $2.97(1.004-8.781)$ & 0.041 \\
\hline Infection with HR-HPV & $2.12(0.78-5.78)$ & 0.133 \\
\hline
\end{tabular}

Table 3: Correlation between an abnormal cervical cytology and any type of HPV infection or HR-HPV infection.

\section{Discussion}

The high prevalence (46.9\%) of overall HPV infection among female university students aged from 18 to 25 years found in the present study is in accordance with rates reported previously. Results of a study conducted in the US by following 608 female university students at six month intervals with HPV testing showed that about $60 \%$ of the women were infected with HPV at some time during the three-year period of the study [23]. Another study from US showed that $44.8 \%$ of women aged 20-24 years were HPV-positive [24]. In Korea, a high prevalence of HPV (38.8\%) was found among 217 sexually active female university students [25]. A recent study using the same HPV genotyping test used in the current study showed a high prevalence (52.1\%) of HPV infection in Greek women less than 25 years of age with genital warts and abnormal cytology [26]. Da Silva, et al. in a study conducted in the State of Paraná (Brazil) showed prevalence of $42.9 \%$ of high-risk HPV, $45.7 \%$ of low risk and $11.7 \%$ unspecified HPV types. Alves, et al. described a prevalence of $88.4 \%$ of HR-HPV in adolescent women, with HPV 16, 51, 31 ad 52 and HPV 18 as the most prevalent types $[27,28]$.

Data on the worldwide prevalence of type-specific HPV have shown a variation in the ranking of HPV types according to geographical regions. However, HPV 16 is the most common HPV type in both single and multiple infections $[4,16]$. Data from the present study showed that HPV 16 was the most frequent type detected in the population analyzed, followed by the types 58,31 and 52. Such data are consistent with the most frequently types detected worldwide, except for HPV 18, which had a low rate in this study [4]. The LR-HPV types 6 and 11 , targeted by the quadrivalent vaccine, were detected with a low prevalence in this group. This observation agrees with the data from a worldwide meta-analysis in which HPV 6 and HPV 11 were the 15th and 18th more detected HPV types [4].

When the students with cytological abnormalities were analyzed as a separate group, a high prevalence of HPV infection was detected. HPV DNA was found in most of the cases reported as ASC-US and in all cases of ASC-H and LSIL. HR-HPV types were present in 9 out of 12 cases of HPV-positive women with abnormal cytologic findings. These data are in agreement with the findings of several studies $[16,29,30]$. Results of a multicenter study involving 3,363 cases of ASCUS tested for $27 \mathrm{HPV}$ types using PGMY09/11 primers showed HPV positivity of 78\% in the 18-24-year age group [29]. In a meta-analysis conducted in 2006, Clifford, et al. reported an overall HPV prevalence rate ranging from $29 \%$ to $100 \%$, with HR-HPV types being detected in most of these cases [16]. Indeed, although the number of the cases was small in the present study, all cytological results of LSIL were HPV-positive and HRHPV genotypes were detected in three-quarters of cases.

Statistical analysis showed an association between the presence of cytological abnormalities and the detection of any HPV type. However, no association was found between cytological abnormalities and infection 
with HR-HPV types. This may be due to the high rates of HR-HPV types in these young women, both with cervical abnormalities and with normal cytological results. In agreement with these findings, data from a metaanalysis showed a high positivity rate of HR-HPV infection in South American women less than 25 years of age [5]. One limitation of the current study, in order to estimate association between HPV infections and cervical abnormalities, might have been the small sample size.

Continued monitoring of HPV infection will be an important strategy for evaluating early impact of the current and the future HPV vaccines, and it may be useful for guiding policies and public health practices. Several clinical trials have shown that the available vaccines are effective against HPV genotypes that cause cervical cancer and genital warts. High-risk HPV types that cause cancer and are covered by both the quadrivalent and bivalent vaccines are HPV 16 and HPV 18. The quadrivalent vaccine also includes protection against HPV types 6 and 11, which are associated with genital warts $[31,32]$.

According Canfell, et al. women living in higher socioeconomic status areas were more likely to be vaccinated against HPV in the catch-up phase of the national program. These authors indicated that although vaccinated women tended to have fewer sexual partners, they also reported prior sexually transmitted disease, which may be a marker of increased risk of prior exposure to HPV and reinforce the continuing need to emphasize equitable delivery of vaccination to various population subgroups [33].

In Brazil, the Ministry of Health introduced in the routine vaccination quadrivalent schedule in 2014 for adolescents of 11-13 years of age. In 2015, the vaccine will be offered to adolescents 9-11 years of age and women with 9-26 years living with HIV. In the first month of HPV vaccination, during which the focus was to mobilize public and private schools across the country, more than 3.4 million girls were immunized against the virus. The number represents $83 \%$ of the goal of the Ministry of Health (MoH). In the second dose the participation of schools was lower and adherence to vaccination dropped considerably, generating lower immunization coverage. More than 2.2 million girls have already received the second dose of the HPV vaccine since the beginning of the new phase of the campaign. The number represents $45 \%$ of 4.9 million girls aged 11 to 13 years [34].

In summary, the high rate of HPV detection in young women emphasizes the advantages of early vaccination. The highest prevalence of minor cytological abnormalities justifies the recommendation of guidelines in relation to cervical cancer screening. In fact, most cytological abnormalities in young women may regress over time, however, it is important consider the possible role of the cumulative influence of infection with single or multiple HPV types in the progression of cervical lesions with increasing age.

\section{Acknowledgments}

This study received a grant from the Foundation for the Support of Research in the State of Goiás (FAPEG). 200810267000192.

\section{References}

1. Baseman J, Koutsky L (2005) The epidemiology of human papillomavirus infections. J Clin Virol 32: 16-24.

2. Wheeler CM (2008) Natural history of human papillomavirus infections, cytologic and histologic abnormalities, and cancer. Obstet Gynecol Clin North Am 35(4): 519-536.

3. Bosch FX, Burchell AN, Schiffman M, Giuliano AR, de Sanjose S, et al. (2008) Epidemiology and natural history of human papillomavirus infections and type-specific implications in cervical neoplasia. Vaccine 26: 1-16.

4. Sanjose S, Diaz M, Castellsagué X, Clifford G, Bruni L, et al. (2007) Worldwide prevalence and genotype distribution of cervical human papillomavirus DNA in women with normal cytology: a , meta-analysis. Lancet Infect Dis 7(7): 453-459.

5. Francesch F, Herrero R, Clifford GM, Snijders PJ, Arslan A, et al. (2006) Variations in the agespecific curves of human papillomavirus prevalence in women worldwide. Int J Cancer 119 (11): 2677 2684.

6. Hoory T, Monie A, Gravitt P, Wu TC (2008) Molecular epidemiology of human papillomavirus. J Formos Med Assoc 107(3): 198-217.

7. Lenselink $\mathrm{CH}$, Melchers WJG, V Quint WG, J Hoebers AM, M Hendriks JC, et al. (2008) Sexual behaviour and HPV infections in 18 to 29 year old women in the pre-vaccine era in the Netherlands. PLoS One 3(11): 3743 .

8. Walboomers JMM, Jacobs MV, Manos MM, Bosch FX, Kummer JA, et al. (1999) Human papillomavírus is a necessary cause of invasive cervical cancer worldwide. J Pathol 189(1): 12-19. 
9. Sanjose S, Quint WG, Alemany L, Geraets DT, Klaustermeier JE, et al. (2010) Human papillomavirus genotype attribution in invasive cervical cancer: a retrospective cross-sectional worldwide study. Lancet Oncol 11(11): 1048-1056.

10. Doorbar J (2005) The papillomavirus life cycle. J Clin Virol 32: 7-15.

11. Woodman CB, Collins SI, Young LS (2007) The natural history of cervical HPV infection: unresolved issues. Nat Rev Cancer 7(1): 11-22.

12. Bernard HU, Burk RD, Chen Z, Koenraad van Doorslaer, Harald zur Hausen, et al. (2010) Classification of papillomaviruses (PVs) based on 189 PV types and proposal of taxonomic amendments. Virology 401(1): 70-79.

13. De Villiers EM, Fauquet C, Broker TR, Bernard HU, zur Hausen $\mathrm{H}$, et al. (2004) Classification of papillomaviruses. Virology 324(1): 17-27.

14. IARC. Internation Agency for Research on Cancer. A review of human carcinogens (2010) - PartB: biological agents. Monographs on the evaluation of carcinogenic risks to humans 100B: 267-295.

15. Ting J, Kruzikas, DT, Smith JS (2010) A global review of age-specific and overall prevalence of cervical lesions. Int J Gynecol Cancer 20(7): 12441249.

16. Clifford G, Franceschi S, Diaz M, Muñoz N, Villa LL (2006) Chapter 3: HPV type-distribution in women with and without cervical neoplastic diseases. Vaccine 24: 26-34.

17. Smith JS, Lindsay L, Hoots B, Keys J, Franceschi S, et al. (2007) Human papillomavirus type distribution in invasive cervical cancer and high-grade cervical lesions: A meta-analysis update. Int J Cancer 121(3): 621-632.

18. Castle PE, Jeronimo J, Schiffman M, Herrero R, Rodríguez AC, et al. (2006) Age-related changes of the cervix influence human papillomavirus type distribution. Cancer Res 66(2): 121.

19. Panatto D, Amicizia D, Trucchi C, Francesca Casabona, Piero Luigi Lai, et al. (2012) Sexual behaviour and risk factors for the acquisition of human papillomavirus infections in young people in Italy: suggestions for future vaccination policies. BMC Public Health 12: 623.
20. Doorbar J, Quint W, Banks L, Bravo IG, Stoler M, at al. (2012) The Biology and Life-Cycle of Human Papillomaviruses. Vaccine 30: 55-70.

21. Tavares SBN, Sousa NLA, Manrique EJC, Pinheiro de Albuquerque ZB, et al. (2011) Improvement in the Routine Screening of Cervical Smears A Study Using Rapid Prescreening and 100\% Rapid Review as Internal Quality Control Methods. Cancer Cytopathol 119(6): 367-376.

22. Solomon D, Nayar R (2005) Bethesda System for Reporting Cervical or Vaginal Cytologic Diagnoses. $2^{\text {nd }}$ (Edn.), Rio de Janeiro: Revinter.

23. Ho GYF, Bierman R, Beardsley L, Chang CJ, Burk RD (1998) Natural history of cervicovaginal papillomavirus infection in young women. $\mathrm{N}$ Engl J Med 338(7): 423-428.

24. Dunne EF, Unger ER, Sternberg M, McQuillan G, Swan DC, et al. (2007) Prevalence of HPV infection among females in the United States. JAMA 297(8): 813-819.

25. Shin HR, Franceschi S, Vaccarella S, Roh JW, Ju YH, et al. (2004) Prevalence and determinants of genital infection with papillomavirus, in female and male university students in Busan, South Korea. J Infect Dis 190(3): 468-476.

26. Papachristou E, Sypsa V, Paraskevis D, Gkekas A, Politi E, et al. (2009) Prevalence of different HPV types and estimation of prognostic risk factors based on the linear array HPV genotyping test. J Med Virol 81(12): 2059-2065.

27. Da Silva MC, Martins HP, De Souza JL, Tognim MC, Svidzinski TI, et al. (2012) Prevalence of HPV infection and genotypes in women with normal cervical cytology in the state of Paraná, Brazil. Arch Gynecol Oncol 286(4): 1015-1022.

28. Alves RRF, Turchi MD, Santos LE, Guimarães EM, Garcia MM, et al. (2013) Prevalence, genotype profile and risk factors for multiple human papillomavirus cervical infection in unimmunized female adolescents in Goiânia, Brazil: a communitybased study. BMC Public Health 13: 1041.

29. Schiffman M, Solomon D. Findings to date from the ASCUS-LSIL Triage Study (ALTS). Arch Pathol Lab Med. 2003; 127: 946-949.

30. Anton G, Peltecu G, Socolov D, Cornitescu F, Bleotu C, et al. (2011) Type-specific human papillomavirus detection in cervical smears in Romania. APMIS 119(1): 1-9. 
31. Harper DM, Franco EL, Wheele C, Ferris DG, Jenkins D, et al. (2004) Efficacy of a bivalent L1 virus-like particle vaccine in prevention of infection with human papillomavirus types 16 and 18 in young women: a randomised controlled trial. Lancet 364(9447): 1757-1765.

32. Villa LL, Costa RL, Petta CA, Andrade RP, Paavonen J, et al. (2006) High sustained efficacy of a prophylactic quadrivalent human papillomavirus types 6/11/16/18 L1 virus-like particle vaccine through 5 years of follow-up. Br J Cancer 95(11): 1459-1466.

33. Canfell K, Egger S, Velentzis LS, Brown JD, O'Connell DL, et al. (2015) Factors related to vaccine uptake by young adult women in the catch-up phase of the National HPV Vaccination Program in Australia: Results from an observational study. Vaccine 33(20): 2387-2394.

34. (2015) Ministry of Health, Department of Health. 